\title{
European Pandemic Influenza Preparedness Planning: A Review of National Plans, July 2016
}

\author{
Maaike Droogers; Massimo Ciotti; Peter Kreidl; Angeliki Melidou; Pasi Penttinen; \\ Chloe Sellwood; Svetla Tsolova; and René Snacken
}

\section{ABSTRACT}

Pandemic influenza A (H1N1) commenced in April 2009. Robust planning and preparedness are needed to minimize the impact of a pandemic. This study aims to review if key elements of pandemic preparedness are included in national plans of European countries. Key elements were identified before and during the evaluations of the 2009 pandemic and are defined in this study by 42 items. These items are used to score a total of 28 publicly available national pandemic influenza plans. We found that plans published before the 2009 influenza pandemic score lower than plans published after the pandemic. Plans from countries with a small population size score significantly lower compared to national plans from countries with a big population $(P<.05)$. We stress that the review of written plans does not reflect the actual preparedness level, as the level of preparedness entails much more than the existence of a plan. However, we do identify areas of improvement for the written plans, such as including aspects on the recovery and transition phase and several opportunities to improve coordination and communication, including a description of the handover of leadership from health to wider sector management and communication activities during the pre-pandemic phase. (Disaster Med Public Health Preparedness. 2019;13:582-592)

Key Words: pandemic influenza, preparedness planning, national preparedness plans, public health preparedness

$\mathrm{I}$ nfluenza pandemics remain among the most critical emergencies to which societies and governments are vulnerable. Robust planning and preparedness are needed to minimize the impact of these events. During the 20th century, 3 influenza pandemics occurred with varying severity and impact, including the 1918 pandemic, which caused approximately 50 million deaths globally. ${ }^{1}$

European national influenza pandemic preparedness plans were assessed ${ }^{2-4}$ before the 2009 influenza pandemic in 2001, 2005, and 2006. In 2001, Paget and Aguilera, on behalf of the European Influenza Surveillance System, reviewed the availability of pandemic plans at national and subnational levels, the availability of a domestic vaccine manufacturer or arrangements for vaccine supply, and the priority groups for vaccination in 16 countries participating in the European Influenza Surveillance System. ${ }^{2}$ In 2006, Mounier-Jack and Coker reviewed the published national pandemic influenza preparedness plans from 21 countries, including European Union (EU) countries, Norway, and Switzerland. At this time a majority of the European population (93\%) was covered by a national pandemic plan, though some issues were not specified in the plans, such as maintenance of essential services, putting plans into practice, and public health interventions. ${ }^{4}$
The awareness of the need of national preparedness plans was heightened following the events surrounding the rapid increase of avian influenza A (H5N1) outbreaks, ${ }^{5}$ with associated human cases from Asia to Europe and Africa after 2005. The need to rapidly respond at an EU level to international events such as A (H5N1) and severe acute respiratory syndrome in 2003 were some of the key arguments for establishing the European Centre for Disease Prevention and Control $^{6,7}$ (ECDC) in 2005. It was only natural that pandemic preparedness was one of the key activities of ECDC during its establishment year and thereafter. Upon a request of the European Council, in 2005 to 2007 ECDC conducted country visits in the EU and European Economic Area (EEA) countries. The objectives of these missions were to update the countries on the current influenza situation, to review the pandemic plans, and to increase the motivation to continue refining the plans. ${ }^{8-10}$

The ECDC developed, in close collaboration with the World Health Organization (WHO) regional office for Europe, self-assessment indicators and tools for member states to ensure that all relevant areas are covered in pandemic planning. ${ }^{11,12}$ These indicators and tools built on previous $\mathrm{WHO}$ guidance on preparedness planning were field-tested during the assessment visits. ${ }^{8,13}$ The 
ECDC self-assessment tool considers national and subnational levels of planning. Key areas covered are planning and coordination, situation monitoring and assessment, prevention and reduction of transmission, health system response, and communication. In September 2006, regional workshops were also organized to stress the importance of cross-sectorial collaboration, business continuity plans, medical countermeasures, and the need for implementation at subnational levels. ${ }^{9}$

The timing of the ECDC country visits from 2005 till 2007 was ideal, as all the visits were conducted before April 2009, when the influenza A (H1N1) pandemic commenced. During the country visits, teams consisting of national key stakeholders involved in preparedness planning, national experts from neighboring countries, and ECDC experts reviewed 30 countries' plans. ${ }^{8-10}$ All countries had developed a plan consistent with international guidance. ${ }^{10}$ Political commitment was perceived and a high level of awareness and motivation was observed in all countries. ${ }^{9} \mathrm{Key}$ areas identified for further work included integrating planning across government sectors and levels, making the plans operational at local level, and an EU-wide assessment of the effectiveness of medical countermeasures. ${ }^{9}$

The response to the 2009 pandemic underlined the need for a coordinated European response regarding availability of vaccines, vaccination strategy, regulatory processes for vaccines, information and communication to the public, global coordination, and multisectorial issues. ${ }^{14}$ These lessons learnt formed the basis of the joint ECDC/WHO guidance for pandemic plan revision. ${ }^{15}$ More specifically on pandemic vaccines, a conference was organized by the Belgian Presidency of the Council of the European Union in March 2010 that identified a number of lessons learnt in the fields of vaccine licensure, prioritization of target groups, importance of communication on pandemic vaccines, implementation of vaccination, and safety monitoring. ${ }^{16}$

Typically, influenza pandemic preparedness is a priority for European countries, as it is considered to be an event of high likelihood with high societal impact. A total of 17 member states shared their national risk assessments with the European Commission in the period of 2011 to 2013, and member states are required to provide the European Commission with summaries of the main elements of their national risk assessments, ${ }^{17}$ again, at the end of 2017.

The objective of this paper is to review the elements of pandemic preparedness in European countries' plans by considering whether key areas for improvement ${ }^{18-20}$ that were identified before and during the evaluations of the 2009 pandemic have been addressed.

\section{METHODS}

A review of 28 national pandemic influenza plans was done for plans from EU and European Free Trade Association countries that were publicly available during July 19-22, 2016. ${ }^{a}$ We assessed whether essential elements, as identified in lessons learnt exercises from the 2009 pandemic, were covered in the plans. A total of 42 items were identified and grouped into 14 overarching themes and a guiding question was developed for each item (Appendix 1). The themes are ordered to represent prepandemic preparedness followed by response aspects and, finally, the recovery phase. Aspects specific to influenza pandemic planning are covered, as well as some generic preparedness activities.

A team of 5 core reviewers analyzed the plans. Support was provided by native language speakers from the ECDC staff to help review those plans in other languages.

\section{Scoring System}

To measure the level of completeness, each plan was scored based on the number of items it covered. Hence, the highest score possible was 42 for a plan that covered all essential elements from the lessons learnt exercises. In order to reflect phraseology used in different languages and countries, the search terms from the item description were expanded to include alternative or synonymous terms. The reviewers scored permissively; for example, if an item was partially met it received a point and an explanation was given to interpret the partial compliance with the item. The items were not weighted and so all 42 items scored equally.

To assess the level of pandemic influenza preparedness at the EU level, the aggregated scores of all plans were used. Hence, this review does not aim to assess individual countries, rather it aims to shed light on the wider, EU level of preparedness.

In addition to reviewing how the plans scored against the 42 -item list, the frequency of the individual items across the 28 plans was also assessed in order to identify specific elements of the plans that were common or not. Hence, "scoring" refers to the general status of the plan or a group of plans, whereas the frequency of an individual item or group of items pinpoints a specific section that was covered by the plan(s).

\section{Stratified Results}

We sought to explain determinants of the plans' scoring by stratifying the results for 4 determinants: publication date, population size, health expenditure, and health management system type. These factors can give a better understanding of the scoring results for groups of countries.

Taking into account that not all national plans were revised after the 2009 pandemic, the plans were categorized as pre2009 and post-2009. Plans published before or during the pandemic are categorized as pre-2009 and the remaining

aECDC, with support of EU/EEA experts and WHO Euro, supported Iceland and Bulgaria in the activities to update the pandemic preparedness plans (in 2016 and 2017). Other countries have also updated their plans since this review was undertaken, and other versions of their documents may be available online. 
plans were categorized as post-2009. This review only considers the most recent version of a country's national plan.

In addition, the results were stratified by population size, ${ }^{21}$ health care expenditure as share of gross domestic product (GDP), ${ }^{22}$ and health management system type. ${ }^{23}$ For population size and health expenditure, countries were grouped in quartiles with the first quartile being the lowest. The data for population size were from 2016, and data for health expenditure were from 2015 or the nearest possible year. For the health management systems, we used the typology published by the European Committee of the Regions, which identifies 4 types of systems: decentralized, partially decentralized, operatively decentralized, and centralized. ${ }^{23}$ This typology was made for all the EU member states in 2011, thereby excluding the European Free Trade Association countries (Norway, Switzerland, and Iceland) and Croatia, which joined the EU later. Countries included in the current study, but missing from the typology, are excluded from these stratified results, as grouping them was outside the scope of the current study. A one-way analysis of variance (ANOVA) was used to test scoring differences between the 4 types of health management systems.

Statistical significance was tested in Microsoft Excel 2011 (version 14.0) with a 2 -tailed $t$ test assuming equal variance and the null hypothesis was rejected when $P<.05$.

4 national plans were not reviewed due to being publicly unavailable (Cyprus, Malta) or legally expired (Lithuania), and Liechtenstein aims to implement the Swiss pandemic plan. Some countries, such as the United Kingdom, have a suite of pandemic preparedness documents, in which case only the main overarching framework or strategy was reviewed. We did not review pandemic plans of subnational organizational structures or from non-health care sectors, unless they were described in the main document.

\section{RESULTS}

Twenty-eight EU/EEA countries have a publicly available pandemic preparedness plan. A majority of these countries' pandemic influenza preparedness plans have not been revised since the 2009 pandemic. Plans published before 2009 were available for 16 countries and plans published after the 2009 pandemic were available for 12 (Figure 1).

The majority of national plans still reflect the 2005 WHO guidance, ${ }^{24}$ including those that have been reviewed following the publication of the updated interim WHO guidance $^{25,26}$ in 2013 and 2017.

In general, there is variation in the scope and approach of the national plans. Plans can be a discussion of strategy, an action plan, or combination of both; some plans are detailed and describe what will be done in response to a pandemic, whereas others are in the style of an action plan and describe what needs to be done to prepare for a pandemic. The plans range from one single document to a suite or an overarching strategy supported by operational documents and annexes, such as a separate communication strategy document. Despite the variation in plans, our methodology worked, as we tested for essential elements that any preparedness plan should include.

Several items were difficult to find in the plans, despite the reviewers knowing that these basic aspects were definitely addressed within national planning arrangements. This occurred when national level planners assumed that business-as-usual processes would be in place (eg, processes for monitoring adverse events or health care capacity). Another reason for not identifying foreseen items was that the information was provided in a separate plan.

\section{Item Frequency and Pinpointing Specific Aspects of the Pandemic Plan}

The frequency of the items in the plans varies with plans' scores, which range from 11 to 40 out of a total of 42 (Figure 2). Items within the themes of "risk communication" and "command, control, coordination, and monitoring" scored the highest and items within the themes "special groups and settings" and "recovery and transition phase" scored the lowest.

An exploration of key elements and themes that received high scores provides an understanding of widely accepted good practices. Nearly all plans (27 out of 28 ) included reference to a national planning committee, often with a description of the composition of the committee and its leadership. Many of the plans (27 out of 28) also refer to a strategy for use of antivirals; however, it was uncommon for the plans to describe a strategy of all the elements of procurement, distribution, storage, and policy. Still, this item received a high score due to our permissive scoring (eg, a plan that only mentions the procurement of antivirals and not all the other aspects listed in the item description would score a point for this item). A communication strategy and ensuring a method to coordinate communication with neighboring countries were both common aspects of the plans ( 27 out of 28 and 26 out of 28 , respectively).

On the other hand, key elements that received low scores suggest thematic areas with less consensus or relevance in the plans. For example, only some (11 out of 28) plans make reference to specific actions for migrants, persons in transit, and hard-toreach populations. Even fewer (10 out of 28) plans describe the roles of organizations in the third sector, with only one plan in particular describing the roles of nongovernmental organizations. Only a few of the plans describe triggers for determining the end of the pandemic (9 out of 28) and the recovery of mobilized human resources ( 7 out of 28 ).

\section{Stratified by Publication Date of Pandemic Influenza Plan (Pre- and Post-2009)}

The comparison of the plans that were published before $(\mathrm{n}=16)$ and after $(\mathrm{n}=12)$ the 2009 influenza pandemic 
Publicly Available Pandemic Influenza Preparedness Plans of 28 European Union / European Free Trade Association Member States in July 2016, Categorized by Plans Pulished Before (Blue) and After (Green) the 2009 Pandemic (map created with mapchart.net). Abbreviations: EU, European Union; EEA, European Economic Area.

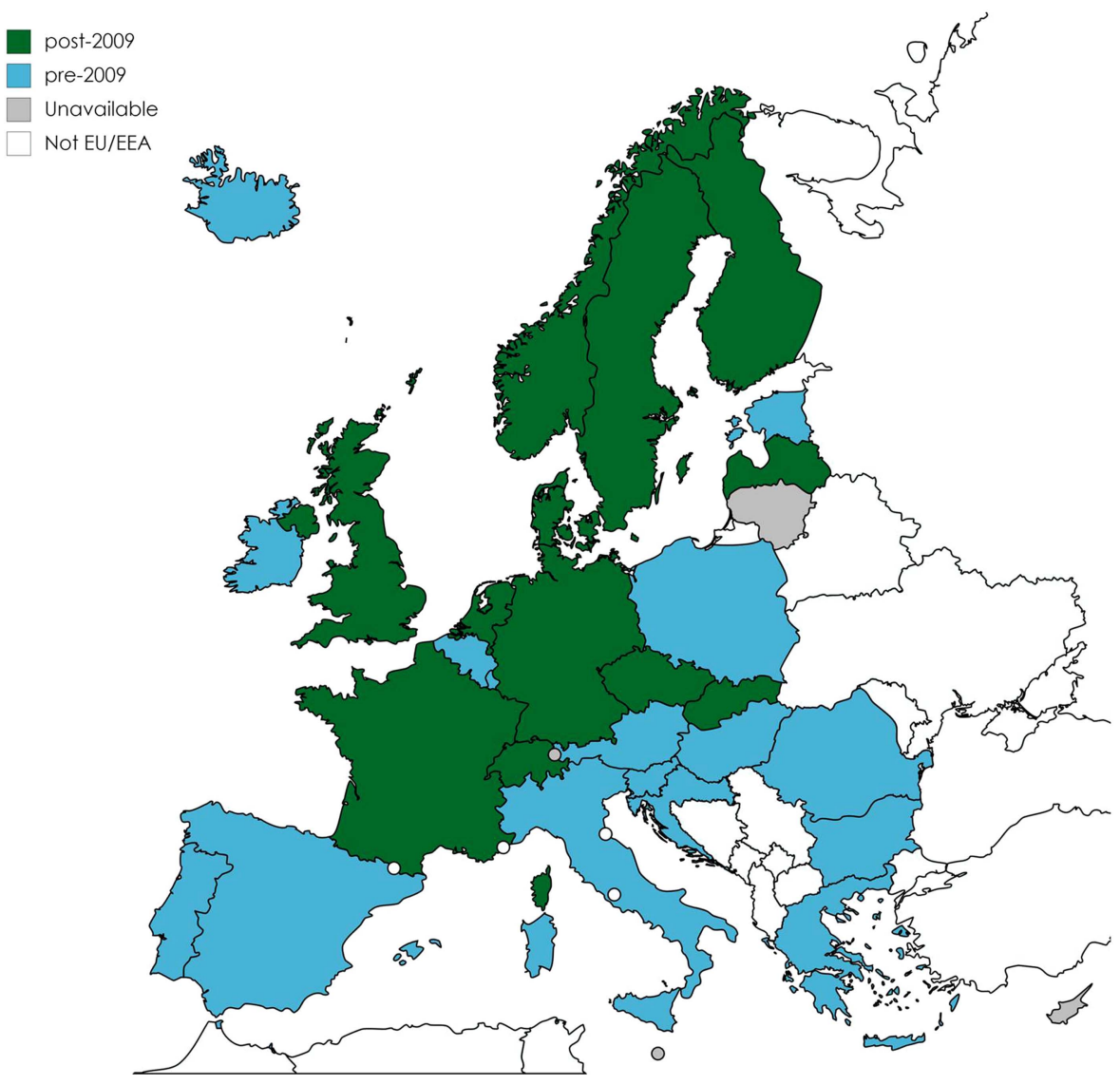

shows that the post-2009 plans received higher scores. For some items ( 9 out of 42 ), the pre-2009 plans received slightly higher scores (items 2, 12, 16, 23, 28, 30, 33, 34, and 36). However, the biggest scoring differences are improvements in the post-2009 plans (Table 1). For example, a methodology to evaluate the pandemic mitigation measures was included in only 5 out of the 16 pre-2009 plans but was included in 10 out of the 12 post-2009 plans.

\section{Effects of Population Size}

National pandemic influenza plans from countries falling within the first quartile in population size (ie, those with the smallest populations) received significantly lower scores compared to those in the other 3 quartiles $(P<.05)$. The scores are determined by the inclusion of the 42 items (see Appendix, Table A1), and on average the plans from the 7 countries with the lowest population size include 20.3 of these items. For the other 3 quartiles, the average score is 32.3 , 32.2, and 30.0, respectively, and they are not significantly different from each other $(P>.05)$ (Figure 3$)$.

\section{Effect of National Health Expenditure}

National health expenditure, expressed as a percentage of the GDP, varies from 5.0 to 11.5 in the countries included in this review. Countries that are at the lower end of this range scored lower than countries with a higher portion of GDP spent on health. This trend can be seen in the scatterplot (Figure 4); however, the correlation is limited $\left(R^{2}=0.12\right)$.

\section{Effect of Health Management System Type}

The management of health systems in the EU member states, including the role of local and regional authorities, has been categorized by the EU and the Committee of the Regions. ${ }^{23}$ According to this categorization there are 4 groups: decentralized, centralized, partially decentralized (ie, countries that have several functions decentralized, but not legislation) and operatively decentralized (ie, countries where local and regional authorities have operational functions in the health management system). Countries with a more centralized health care system appear to have more comprehensive pandemic plans, while countries with a decentralized health management system 
FIGURE 2

Key elements $(\mathrm{N}=42)$ of a national pandemic influenza preparedness plan, including frequency in the 28 reviewed plans. The highest-scoring items are highlighted in green and the lowest-scoring items are highlighted in red. For a description of the items, see Appendix, Table A1.

\begin{tabular}{|c|c|c|c|}
\hline Theme & Item & $\begin{array}{l}\text { Frequency of Item in Plans } \\
\qquad(n=28)\end{array}$ & $\begin{array}{c}\text { Average } \\
\text { perTheme }\end{array}$ \\
\hline \multirow{3}{*}{ Preparedness planning } & 1 Simulation exercises & 17 & \multirow{3}{*}{20} \\
\hline & 2 National planning committee & 27 & \\
\hline & 3 Evaluation methodology & 15 & \\
\hline \multirow{3}{*}{ Strategic planning } & 4 Activation/de-escalation triggers & 17 & \multirow{3}{*}{18} \\
\hline & 5 Ethical aspects & 19 & \\
\hline & 6 Planning assumptions & 18 & \\
\hline \multirow{3}{*}{ Risk-based planning } & 7 Risk assessment capacity & 16 & \multirow{3}{*}{21} \\
\hline & 8 National surveillance and monitoring & 25 & \\
\hline & 9 Differentiated response planning & 21 & \\
\hline \multirow{3}{*}{$\begin{array}{l}\text { Command control, } \\
\text { coordination, \& monitoring }\end{array}$} & 10 Crisis management system & 23 & \multirow{3}{*}{23} \\
\hline & 11 Decision-making data requirements & 21 & \\
\hline & 12 Technical advice for decision-making links & 24 & \\
\hline \multirow{3}{*}{ Risk communication } & 13 Communications strategy & 27 & \multirow{3}{*}{26} \\
\hline & 14 Communication channels & 25 & \\
\hline & 15 Cross-border communication coordination & 26 & \\
\hline \multirow{4}{*}{$\begin{array}{l}\text { Early warning, risk } \\
\text { assessment, \& surveillance }\end{array}$} & 16 Investigation of first cases & 19 & \multirow{4}{*}{20} \\
\hline & 17 Routine seasonal surveillance & 24 & \\
\hline & 18 Surveillance feedback & 19 & \\
\hline & 19 Adaptable laboratory surveillance & 19 & \\
\hline \multirow{3}{*}{ Vaccines } & 20 Vaccine policy & 24 & \multirow{3}{*}{19} \\
\hline & 21 Vaccine uptake and adverse events & 19 & \\
\hline & 22 Vaccine effectiveness & 13 & \\
\hline \multirow{3}{*}{$\begin{array}{l}\text { Antivirals \& other essential } \\
\text { medicines }\end{array}$} & 23 Antiviral policy & 27 & \multirow{3}{*}{21} \\
\hline & 24 Antiviral adverse events & 16 & \\
\hline & 25 Other essential medicines & 20 & \\
\hline \multirow{3}{*}{$\begin{array}{l}\text { Health care system } \\
\text { preparedness and response }\end{array}$} & 26 Existing health care capacity awareness & 21 & \multirow{3}{*}{21} \\
\hline & 27 Surge capacity & 25 & \\
\hline & 28 Healthcare information exchange & 16 & \\
\hline \multirow{2}{*}{$\begin{array}{l}\text { Nonpharmaceutical public } \\
\text { health interventions (NPIs) }\end{array}$} & 29 NPI policy & 23 & \multirow{2}{*}{21} \\
\hline & $30 \mathrm{NPI}$ communication strategy & 19 & \\
\hline \multirow{3}{*}{$\begin{array}{l}\text { Essential services \& } \\
\text { business continuity }\end{array}$} & 31 Essential services identification & 14 & \multirow{3}{*}{16} \\
\hline & 32 Health business continuity & 19 & \\
\hline & 33 Vulnerable group support & 15 & \\
\hline \multirow{3}{*}{ Special groups and settings } & 34 Business and workplace preparedness & 17 & \multirow{3}{*}{13} \\
\hline & 35 Vulnerable populations & 11 & \\
\hline & 36 Third sector engagement & 10 & \\
\hline \multirow{3}{*}{$\begin{array}{l}\text { Recovery and transition } \\
\text { phase }\end{array}$} & 37 Recovery and transition & 18 & \multirow{3}{*}{11} \\
\hline & 38 Triggers for recovery & 9 & \\
\hline & 39 Human resources recovery & 7 & \\
\hline & 40 International Health Regulations core capacit & 18 & \\
\hline International & 41 Interoperability with neighbors & 17 & 19 \\
\hline interoperability & 42 International communication & 23 & \\
\hline
\end{tabular}

\section{Essential Elements in the Pandemic Influenza Preparedness Plans With the Largest Difference in Frequency of Mentions} Between the Post-2009 and Pre-2009 Plans

Theme

Item

Preparedness

planning

Recovery and

transition phase

Strategic

planning

Vaccines

\section{Evaluation} methodology

37 Recovery and transition

5 Ethical aspects

21 Vaccine uptake and adverse events

\section{Description}

The plan includes a methodology to evaluate the pandemic mitigation measures.

The plan refers to development of risk-based triggers to identify the end of the pandemic and trigger recovery.

The plan discusses and describes ethical aspects of mitigation measures.

The plan refers to a system for monitoring vaccine uptake and adverse events.
Pre-2009 Plans

$(n=16)$, No. $(\%)$

Post-2009 Plans

$5(31 \%)$

$10(83 \%)$

$7(44 \%)$

$11(92 \%)$

$8(50 \%)$

$11(92 \%)$

$8(50 \%)$

$11(92 \%)$ 


\section{FIGURE 3}

\section{Scores of National Pandemic Influenza Plans $(\mathrm{N}=28)$ for Inclusion of Essential Elements, Stratified by Quartiles of Population Size}

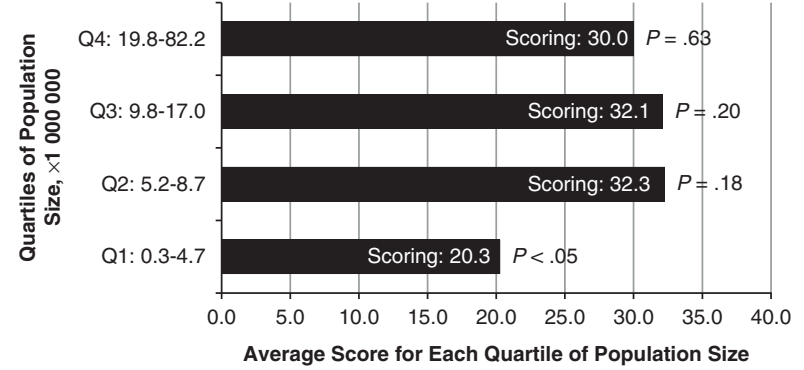

Q1: Croatia, Estonia, Iceland, Ireland, Latvia, Luxembourg, Slovenia.

Q2: Austria, Bulgaria, Denmark, Finland, Norway, Slovakia, Switzerland.

Q3: Belgium, Czech Republic, Greece, Hungary, Netherlands, Portugal, Sweden Q4: France, Germany, Italy, Poland, Romania, Spain, United Kingdom.

\section{FIGURE 4}

\section{Correlation Between National Health Expenditure and National Pandemic Influenza Plan Scores in European Countries $(\mathrm{N}=28)$}

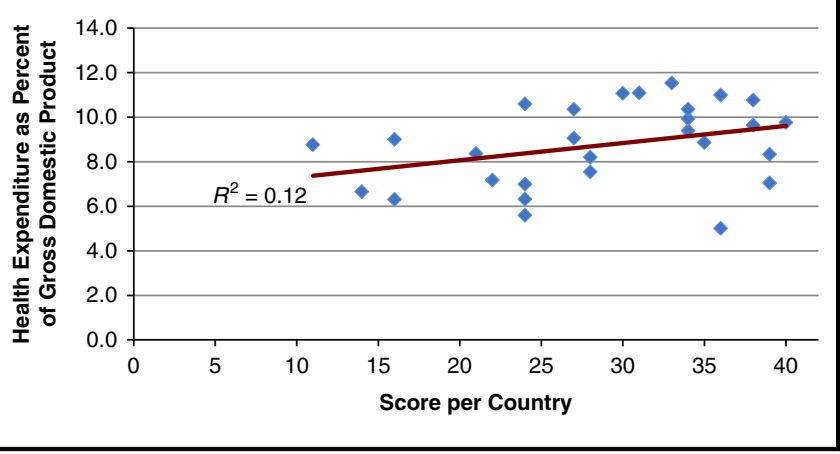

score lower (Table 2). It could be relevant that the 3 countries categorized as having decentralized health management systems do not have publicly available post-2009 pandemic plans. Testing for the differences of the means of the 4 health management system types with a one-way analysis of variance (ANOVA) gives a nonsignificant result $(P=.57)$.

\section{DISCUSSION AND CONCLUSIONS}

Following the evaluation of the 2009 pandemic, the legal framework Decision No. 1082/2013/EU on serious crossborder threats to health has been adopted in the EU. ${ }^{27,28}$ This framework has a broader perspective on preparedness and response to public health emergencies, highlighting the all-hazard approach, including a joint procurement procedure for the purchase of medical countermeasures for serious crossborder threats to health. There is no public mapping of European Member States' compliance with this legislative framework. $^{29}$

\section{TABLE 2}

\section{Frequency of Essential Elements in National Pandemic Influenza Plans Stratified by Type of Health Management System in Country}

Health Management System Type ${ }^{a}$ Number of Countries Average Score

Decentralized

$\mathrm{n}=3^{\mathrm{b}}$

25.7

Partially decentralized

Operatively decentralized

$n=9^{c}$

$n=8^{d}$

$n=4^{e}$

28.6

30.5

33.3

${ }^{a}$ According to categorization by the European Committee of the Regions. ${ }^{24}$

${ }^{\mathrm{b}}$ Austria, Italy, Spain

${ }^{\mathrm{C}}$ Belgium, Czech Republic, Denmark, Estonia, Finland, Germany,

Hungary, Poland, Sweden

dBulgaria, Latvia, Luxembourg, Netherlands, Romania, Slovakia, Slovenia, United Kingdom

${ }^{\mathrm{e}}$ France, Greece, Portugal, Ireland

The legal framework on serious cross-border threats to health ${ }^{30}$ requires European countries to report every 3 years on preparedness planning, ${ }^{31}$ including International Health Regulations implementation, business continuity planning, and interoperability of plans between health and other sectors, as well as international collaboration in case of public health emergency. ${ }^{32}$ Influenza pandemics remain the most likely pandemic threat, with very serious health and socioeconomic consequences. ${ }^{33}$

The current review takes forward the work on the pandemic plans by describing the gaps, where the lessons learned ${ }^{14,16,18-20}$ from the influenza A (H1N1) pandemic in 2009 remain to be included in the plans. We realize that plans are continuously being revised. For this current review we used the plans that were publicly available on the website of the country's responsible authority in July 2016. The most recent versions of the plans can be found on the ECDC website. ${ }^{34}$

Reflecting on the available pandemic influenza preparedness plans shows 2 thematic areas that were mentioned least often in the plans: elements related to the recovery and transition phase, and special groups and settings. These are opportunities for improvement. Public health preparedness for special groups and vulnerable groups are elements not specific for pandemic preparedness and countries might have provisions for health care services to these groups in other legislative or strategic frameworks. The role of these vulnerable groups was also highlighted as a component of community preparedness. ${ }^{35}$ We note that these areas for improvement did not qualify among the 10 most urgent recommendations in a recent survey of EU preparedness experts. ${ }^{36}$

In addition, we found several opportunities for improvement related to coordination and communication. The handover of leadership from health to wider sector management was often unclear, even though this is important to ensure leadership 
continuity and a smooth response. Another opportunity for improvement we found is that few plans identified communication activities during the prepandemic phase, despite this being important to ensure all parties are aware of work underway and know the shared planning assumptions. A third issue is that while some plans allude to increasing health care capacity, very few describe the process to understand the baseline, though this may be covered in other documentation. This knowledge on the current status quo is essential to a successful response. Tools are available for countries to assess their preparedness and response capabilities. ${ }^{37,38}$

This manuscript identifies a number of planning deficits that remain in the national pandemic preparedness plans reviewed. As highlighted before, there are some common deficits; however, each plan has its own specific shortcomings. Countries planning to update their plan might want to prioritize which aspects to update first. To prioritize, one can reflect on the pandemic preparedness planning cycle that consists of 5 key elements of preparedness planning. ${ }^{15}$ These 5 key elements can provide guidance in prioritizing efforts to update pandemic plans. The prioritization provided by outbreak preparedness experts can also be helpful. ${ }^{36}$

Plans are organized in various manners, reflecting perhaps also the differences in framing the pandemic narrative as described by Holmberg and Lundgren. ${ }^{39}$ One specific manner of organizing the plan is not better; rather, in order to be effective, pandemic influenza preparedness must fit within existing overarching allhazard plans, the national legal framework, and planning arrangements, and must serve well the existing organizational structures. From an EU perspective, the divergence in national plans could be seen as a challenge when wishing to coordinate across member states. ${ }^{39}$ In the current review we considered interoperability nationally and internationally as one of the essential elements that should be taken under consideration.

Countries face different challenges in the implementation and coordination of pandemic preparedness plans, which are likely due to regionalized decision-making in some countries (eg, federal states and autonomous regions). This is suggested by the fact that the few countries with decentralized health care structures scored slightly lower on average than other countries. A decentralized structure may require a lot of negotiations and consultations with local authorities as well as resolving issues of ownership and funding (including negotiations regarding human resources for the preparedness planning versus response). However, decentralization could also lead to having a hierarchical structure of emergency plans, whereas national or federal plans are strategic, leaving operational and tactical plans to the regions. The need for intersectorial collaboration for essential services and capacities can further complicate preparedness activities.

In 2005, when the bulk of pandemic preparedness was undertaken, many countries were of the view that stand-alone pandemic influenza plans were needed. Experiences from the 2009 pandemic and with other hazards (eg, Ebola, Middle East Respiratory Syndrome Coronavirus) have demonstrated the benefit of aligning plans for response to pandemic influenza more closely to existing arrangements that are used on a more frequent basis. An all-hazards approach is also taken on by the WHO interim guidance for pandemic influenza risk management. ${ }^{26}$ This ensures that responders are familiar with response arrangements and that the best outcomes should be achieved for patients and the public. Whilst a pandemic is not the same as a seasonal outbreak of influenza or common situations experienced during winter due to cold weather, such as norovirus circulation, there is certainly learning from both of these scenarios that can be built upon to ensure robust pandemic response.

Our results suggest that the limited resources available for planning at national level, due to the size of the country or low health expenditure, appear to be factors influencing the contents of the pandemic plans (Figures 3 and 4). One can argue that a small country does not need to have the same level of complexity in the plans as a larger country does due to simpler structures and planning and response processes in the country. It might be interesting to investigate if the plans have been supported by financial mechanisms for funding specific activities; however, this was beyond the scope of the current study.

The plans published after 2009 appear to have included more of the identified essential elements than those published before 2009. We suggest this is due to the lessons learnt and evaluation process undertaken in countries and at the EU and global levels and is part of a continuously evolving planning process. Similar results suggesting temporal improvement of plans were shown by Moen and colleagues ${ }^{40}$ among 40 countries between 2008 and 2010. However, it should be noted that in our analysis we are not comparing pre- and post-pandemic plans originating from the same countries. It is likely that the countries that have had the resources to update their plans will also be more comprehensive in their plans and they may have had a more comprehensive plan prior to the post-2009 revision.

It was expected that plans updated and published following the 2009 pandemic would contain more of the key elements than those written before the pandemic. However, conversely, 9 elements are more common in plans written before the 2009 pandemic. This may be because post-2009 pandemic response arrangements are spread over numerous documents that have not been included in the analysis. Another clarification could be that post-2009 plans follow the older WHO 2005 planning guidance.

We stress that the review of written plans does not reflect the actual preparedness level, as the level of preparedness entails much more than the existence of a plan. Also, Meeyai and colleagues $^{41}$ did not find that the completeness of plans in 19 
European countries was associated with measures of the pandemic intensity during 2009. We recognize that the process of developing the plan is often as important as the resulting published documentation, or even more. As for the review of the written plans, we note some limitations. The level of detail per item was not assessed. However, the level of detail of a plan is probably less important than the completeness of a plan (ie, covering all essential elements), as flexible plans are needed to respond to the next pandemic of which the parameters are unknown. ${ }^{42}$ We also point out that our baseline is the WHO guidance from 2009, ${ }^{13}$ which could clarify why countries pre-2009 scored lower in this review.

The authors are aware that a lot of activities take place at different levels in Europe with regards to pandemic preparedness, such as simulation exercises and after action reviews. Also, the Joint External Evaluations that have a much wider scope than pandemics are recommended as part of the international health regulations to incorporate-in addition to self-assessment-also a peer review with domestic and external experts. Indeed, these activities increase the capacity to respond, but it is difficult to obtain evidence on these activities until lessons learned are published. Therefore, we recommend for lessons learned and good practices to be exchanged more frequently among key stakeholders.

\section{About the Authors}

European Public Health Association, Utrecht, Netherlands (Dr Droogers), European Centre for Disease Prevention and Control, Stockholm, Sweden (Drs Droogers, Ciotti, Melidou, Penttinen, Tsolova, and Snacken), Department of Hygiene, Medical Microbiology and Public Health, Medical University Innsbruck, Austria (Dr Kreidl) and National Health Service England, London, United Kingdom (Dr Sellwood).

Correspondence and reprint requests to Maaike Droogers, Otterstraat 118, 3513CR, Utrecht, Netherlands (e-mail: maaikedroogers@gmail.com).

\section{Acknowledgments}

The authors would like to thank the colleagues at the European Centre for Disease Prevention and Control (ECDC) for helping translate the national pandemic influenza preparedness plans: Kaja Kaasik Aaslav, Agne Bajoriniene, Dragoslav Domanovic, Katrin Leitmeyer, Otilia Mårdh, Paulina Nakielny, Diamantis Plachouras, Vladimir Prikazsky, and Signe Rirdance.

\section{REFERENCES}

1. Johnson NP, Mueller J. Updating the accounts: global mortality of the 1918-1920 "Spanish" influenza pandemic. Bull Hist Med. 2002;76 (1):105-115.

2. Paget WJ, Aguilera JF. Influenza pandemic planning in Europe. Euro surveillance: Bulletin européen sur les maladies transmissibles. 2001;6 (9):136-140.

3. Ciotti M, Karcher F, Ganter B, et al. Results of survey of national influenza pandemic preparedness in Europe. Euro surveillance: Bulletin européen sur les maladies transmissibles. 2005;10(3):E050303.1.
4. Mounier-Jack S, Coker RJ. How prepared is Europe for pandemic influenza? Analysis of national plans. Lancet. 2006;367(9520):1405-1411.

5. Snacken R, Kendal AP, Haaheim LR, et al. The next influenza pandemic: lessons from Hong Kong, 1997. Emerg Infect Dis. 1999;5 (2):195-203

6. European Commission. Regulation (EC) No 851/2004 of the European Parliament and of the Council Establishing a European Centre for Disease Prevention and Control. Official Journal of the European Union; 2004.

7. Greer S. The European Centre for Disease Prevention and Control: hub or hollow core? J Health Polit Policy Law. 2012;23(8):1813817.

8. Nicoll A, Kreidl P. Preparing the European Union for the next pandemic - half way there. Euro surveillance: Bulletin européen sur les maladies transmissibles. 2007;12(12):E071220.5.

9. European Centre for Disease Prevention and Control. Technical report: Pandemic Influenza Preparedness in the EU. Status Report as of Autumn 2006. http://ecdc.europa.eu/en/publications/publications/0701_ter_pan demic_influenza_preparedness.pdf. Published 2007.

10. European Centre for Disease Prevention and Control. Technical Report: Pandemic Influenza Preparedness in the EU/EEA. Status Report as of Autumn 2007. http://ecdc.europa.eu/en/publications/Publications/0712_ TER_Pandemic_Influenza_Preparedness_in_EU_EEA.pdf. Published 2007.

11. European Centre for Disease Prevention and Control. Assessment Tool for Influenza Preparedness in European Countries - With a Main Focus on Pandemic Preparedness. http://ecdc.europa.eu/en/healthtopics/Docu ments/0705_Pandemic_Influenza_Preparedness_Indicators.pdf. Published 2007.

12. World Health Organization. Joint European Pandemic Preparedness Self Assessment Indicators http://ecdc.europa.eu/en/publications/publica tions/100326_joint_european_pandemic_indicators.pdf. Published 2010.

13. World Health Organization. Pandemic Influenza Preparedness and Response. WHO guidance document. http://apps.who.int/iris/bitstream/ 10665/44123/1/9789241547680_eng.pdf. Published 2009.

14. Council of the European Union. Council Conclusions on Pandemic (H1N1) 2009 - a Strategic Approach: 2965th Employment, Social Policy, Health and Consumer Affairs Council Meeting. http://ec.europa. eu/health/ph_overview/health_forum/docs/ev_20091016_rd13_en.pdf. Published 2009.

15. European Centre for Disease Prevention and Control. Guide to Revision of National Pandemic Influenza Preparedness Plans - Lessons Learned From the 2009 A(H1N1) Pandemic. Stockholm: European Centre for Disease Prevention and Control; 2017.

16. Hanquet $G$, Van Damme P, Brasseur D, et al. Lessons learnt from pandemic A(H1N1) 2009 influenza vaccination: highlights of a European workshop in Brussels (22 March 2010). Vaccine. 2011;29(3):370-377.

17. European Commission. The Post 2015 Hyogo Framework for Action: Managing Risks to Achieve Resilience. http://ec.europa.eu/echo/files/ news/post_hyogo_managing_risks_en.pdf. Published 2014.

18. World Health Organization. Key Changes to Pandemic Plans by Member States of the WHO European Region Based on Lessons Learnt From the 2009 Pandemic. http://www.euro.who.int/_data/assets/pdf_file/0006/ 161664/ECDC_WHO_EURO_PiP-Workshops-Summary-Report_FINAL26032012.pdf. Published 2012. Accessed February 4, 2018.

19. Nicoll A, Brown C, Karcher F, et al. Developing pandemic preparedness in Europe in the 21st century: experience, evolution and next steps. Bull World Health Organ. 2012;90(4):311-317.

20. European Centre for Disease Prevention and Control. The 2009 A (H1N1) Pandemic in Europe - A Review of the Experience. https://ecdc. europa.eu/sites/portal/files/media/en/publications/Publications/101108_ SPR_pandemic_experience.pdf. Published 2010. Accessed February 4, 2018.

21. Eurostat. http://ec.europa.eu/eurostat/tgm/table.do?tab=table\&plugin= $1 \&$ language $=$ en $\&$ pcode $=$ tps00001. Published 2016.

22. OECD / European Union. Health at a Glance: Europe 2016 - State of Health in the EU Cycle. Paris: The Organisation for Economic Cooperation and Development Publishing; 2016. 
23. European Union. The Management of Health Systems in the EU Member States - The Role of Local and Regional Authorities. http://cor. europa.eu/en/documentation/studies/Documents/health-systems/health-sys tems-en.pdf. Published 2012.

24. World Health Organization. WHO Global Influenza Preparedness Plan: The Role of WHO and Recommendations for National Measures Before and During Pandemics. http://www.who.int/csr/resources/publications/ influenza/WHO_CDS_CSR_GIP_2005_5.pdf. Published 2005.

25. World Health Organization. Pandemic Influenza Risk Management, WHO Interim Guidance. Guidance. http://www.who.int/influenza/pre paredness/pandemic/GIP_PandemicInfluenzaRiskManagementInterimGui dance_2013.pdf. Published 2013.

26. World Health Organization. Pandemic Influenza Risk Management WHO Guidance. http://www.who.int/influenza/preparedness/pandemic/ PIRM_withCoverPage_201707_FINAL.pdf?ua=1. Published 2017.

27. European Commission. Report From the Commission to the European Parliament and the Council: Report on the Implementation of Decision No 1082/2013/EU of the European Parliament and of the Council of 22 October 2013 on Serious Cross-Border Threats to Health and Repealing Decision No 2119/98/EC. http://ec.europa.eu/health//sites/health/files/ preparedness_response/docs/report_decision_serious_crossborder_threats_ 22102013_en.pdf. Published 2015.

28. The European Parliament Council of the European Union. Decision No 1082/ 2013/EU of the European Parliament and of the Council of 22 October 2013 on Serious Cross-Border Threats to Health and Repealing Decision No 2119/ 98/EC 2013. http://ec.europa.eu/health//sites/health/files/preparedness_ response/docs/decision_serious_crossborder_threats_22102013_en.pdf. Accessed February 4, 2018.

29. Speakman EM, Burris S, Coker R. Pandemic legislation in the European Union: fit for purpose? The need for a systematic comparison of national laws. Health Policy. 2017;121(10):1021-1024.

30. World Health Organization. International Health Regulations. http:// apps.who.int/iris/bitstream/10665/43883/1/9789241580410_eng.pdf. Published 2005.

31. European Commission. Implementing Decision No 1082/2013/EU of the European Parliament and of the Council With Regard to the Template for Providing the Information on Preparedness and Response Planning in Relation to Serious Cross-Border Threats to Health. http://eur-lex. europa.eu/legal-content/EN/TXT/PDF/?uri=CELEX:32014D0504\&from $=$ EN. Published 2014. Accessed February 4, 2018.
32. European Commission. Report on the Implementation of Decision No $1082 / 2013 / \mathrm{EU}$ of the European Parliament and of the Council of 22 October 2013 on Serious Cross-Border Threats to Health and Repealing Decision No 2119/98/EC. https://ec.europa.eu/health/sites/ health/files/preparedness_response/docs/report_decision_serious_crossborder_threats_22102013_en.pdf. Published 2015. Accessed February 4, 2018.

33. Saunders-Hastings P, Reisman J, Krewski D. Assessing the State of Knowledge Regarding the Effectiveness of Interventions to Contain Pandemic Influenza Transmission: A Systematic Review and Narrative Synthesis. PLoS One. 2016;11(12):e0168262.

34. European Centre for Disease Prevention and Control. Influenza Pandemic Preparedness Plans. https://ecdc.europa.eu/en/seasonal-influ enza/preparedness/influenza-pandemic-preparedness-plans. Published 2017. Accessed June 15, 2018.

35. Ramsbottom A, O'Brien E, Ciotti L, et al. Enablers and barriers to community engagement in public health emergency preparedness. J Community Health. 2017;43(2):1-9.

36. Belfroid E, Timen A, van Steenbergen JE, et al. Which recommendations are considered essential for outbreak preparedness by first responders? BMC Infect Dis. 2017;17(1):195.

37. MacDonald G, Moen AC, St Louis ME. The national inventory of core capabilities for pandemic influenza preparedness and response: an instrument for planning and evaluation. Influenza Other Respir Viruses. 2014;8(2):189-193.

38. World Health Organization. Joint External Evaluation Tool: International Health Regulations. http://apps.who.int/iris/bitstream/10665/ 204368/1/9789241510172_eng.pdf. Published 2005.

39. Holmberg M, Lundgren B. Framing post-pandemic preparedness: comparing eight European plans. Glob Public Health. 2016:1-16.

40. Moen A, Kennedy PJ, Cheng PY, et al. National inventory of core capabilities for pandemic influenza preparedness and response: results from 36 countries with reviews in 2008 and 2010. Influenza Other Respir Viruses. 2014;8(2):201-208.

41. Meeyai A, Cooper BS, Coker R. Analysis of 2009 pandemic influenza A/ H1N1 outcomes in 19 European countries: association with completeness of national strategic plans. BMJ Open. 2013;3(3).

42. Napoli C, Fabiani M, Rizzo C, et al. Assessment of human influenza pandemic scenarios in Europe. Euro surveillance: bulletin Europeen sur les maladies transmissibles. 2015;20(7):29-38. 
TABLE A1

Key Elements of a National Pandemic Influenza Preparedness Plan (42 Items).

\section{Theme}

Preparedness planning

Strategic planning

Risk-based planning

Command control, coordination \& monitoring

Risk communication

Early warning, risk assessment \& surveillance

Vaccines

Antivirals \& other essential medicines

Health care system

preparedness and response

Nonpharmaceutical public health interventions (NPIs)

Essential services \& business continuity

Special groups and settings

\section{Item}

1 Simulation exercises

2 National planning committee

3 Evaluation methodology

4 Activation/de-escalation triggers

5 Ethical aspects

6 Planning assumptions

7 Risk assessment capacity

8 National surveillance and monitoring

9 Differentiated response planning

10 Crisis management system

11 Decision-making data requirements

12 Technical advice for decisionmaking links

13 Communications strategy

14 Communication channels

15 Cross-border communication coordination

16 Investigation of first cases

17 Routine seasonal surveillance

18 Surveillance feedback

19 Adaptable laboratory surveillance

20 Vaccine policy

21 Vaccine uptake and adverse events

22 Vaccine effectiveness

23 Antiviral policy

24 Antiviral adverse events

25 Other essential medicines

26 Existing health care capacity awareness

27 Surge capacity

28 Health care information exchange

29 NPI policy

$30 \mathrm{NPI}$ communication strategy

31 Essential services dentification

32 Health business continuity

33 Vulnerable group support

34 Business and workplace preparedness

35 Vulnerable populations

36 Third sector engagement

\section{Description}

The plan refers to simulation exercises.

The plan refers to a national planning/preparedness committee.

The plan includes a methodology to evaluate the pandemic mitigation measures.

The plan refers to defined, country-specific triggers for activation and de-escalation of mitigation measures.

The plan discusses and describes ethical aspects of mitigation measures.

The plan includes a range of realistic, country-specific planning assumptions.

The plan refers to capacity and processes to perform national and subnational risk assessments.

The plan refers to a national surveillance system to collect and analyze epidemiological and virological data (eg, virology, risk groups, transmission, clinical severity, vaccination uptake, antiviral consumption).

The plan considers the possibility of differentiated responses depending on the situation severity.

The plan describes activation of health sector and multisectorial crisis management systems.

The plan defines information requirements for decision-making.

The plan describes the crisis management system links to technical and decision-making levels.

The plan describes the need for a communication strategy for the preparedness and response phases

The plan refers to strategies for targeting pandemic communications to different groups.

The plan describes methods to coordinate communication with neighboring countries (eg, the Early Warning and Response System of the European Union).

The plan specifies the approach for rapid investigation of the first indigenous cases.

The plan refers to the availability of sustainable, routine seasonal influenza surveillance that can be adapted to pandemic requirements.

The plan describes the processes to provide feedback to surveillance stakeholders and others.

The plan describes differential laboratory testing depending on the situation severity (eg, decreasing testing as the pandemic evolves).

The plan refers to a strategy for use of pandemic vaccine (eg, procurement, distribution, storage, and policy).

The plan refers to a system for monitoring vaccine uptake and adverse events.

The plan identifies a mechanism to monitor pandemic vaccine effectiveness.

The plan refers to a strategy for use of antivirals during a pandemic (eg, procurement, distribution, storage, and policy)

The plan describes a system for rapid detection and monitoring of pharmaceutical adverse events or side effects.

The plan foresees an increased need for other medicines (eg, antibiotics, nonsteroida antiinflammatory drugs).

The plan identifies the need for awareness of health care capacities at the time of activation.

The plan describes surge capacity for laboratory tests and patient care

The plan proposes establishment of a network of clinicians, nurses, public health authorities, and health care authorities for frequent and rapid information exchange.

The plan refers to a policy describing the relevant NPIs.

The plan refers to a strategy to communicate NPIs to the public and other target groups.

The plan identifies essential public and private services.

The plan requires the ministry of health, key public health agencies, and major health facilities to have business continuity plans.

The plan refers to identified methodologies to support vulnerable groups (eg, at-risk patient groups).

The plan refers to preparedness/business continuity for businesses and workplaces.

The plan refers to specific actions for migrants, persons in transit, and hard-to-reach populations.

The plan describes roles for third sector organizations: nongovernmental organizations volunteer organizations, or community-based organizations (eg, Red Cross and aid organizations). 
European Pandemic Influenza Preparedness Plans

\section{TABLE A1}

\section{(Continued)}

Theme

Item

Recovery and transition phase 37 Recovery and transition

38 Triggers for recovery

International interoperability
39 Human resources recovery

40 International health regulations core capacity

42 International communication

\section{Description}

The plan describes recovery and transition activities.

The plan refers to development of risk-based triggers to identify the end of the pandemic and trigger recovery.

The plan outlines the recovery for staff and responders mobilized during a pandemic.

The plan cross references with international health regulations for core capacity requirements.

The plan describes requirements for interoperability of plans with neighboring countries.

The plan outlines communication strategies with international organizations. 